\title{
The Effects of Lead Exposure on the Activities of $\delta$-Aminolevulinic Acid Dehydratase with the Modification of the Relative Genotypes
}

\author{
Y.-W. Chiu ${ }^{1}$, T.-Y. Liu ${ }^{2}$ and H.-Y. Chuang ${ }^{3}$ \\ ${ }^{1}$ Department of Family Medicine, Ta-Tung Municipal Hospital, and Faculty of Medicine, Kaohsiung Medical \\ University, Kaohsiung 807, TAIWAN, cyw6328@yahoo.com.tw \\ ${ }^{2}$ Graduate Institute of Occupational Safety and Health, Kaohsiung Medical University, Kaohsiung 807, TAIWAN, \\ chy@seed.net.tw \\ ${ }^{3}$ Department of Community Medicine, Kaohsiung Medical University Hospital, and Department of Public Health, \\ College of Health Science Kaohsiung Medical University, Kaohsiung 807, TAIWAN, ericch@kmu.edu.tw
}

\begin{abstract}
To investigate the effects of blood lead and other related factors on $\delta$-aminolevulinic acid dehydratase (ALAD) activity in lead workers. In 121 lead workers and 117 reference subjects, the following data were collected from health examination: blood lead, BMI, glucose AC, and Hct. A questionnaire including of demographic data, medical history, smoking and alcohol consumption was completed by each of subjects. ALAD activity was determined by the standardized method of the European Community. ALAD genotyping was using a method of PCR-RFLP. In this study, 229 ALAD1-1 homozygotes (96.2\%), 8 ALAD1-2 heterozygotes (3.8\%) were identified, and none of ALAD2-2 homozygote was observed. Blood lead levels in lead workers and reference subjects were $19.5 \mu \mathrm{g} / \mathrm{dL}(\mathrm{SD}=14.7)$ and $2.9 \mu \mathrm{g} / \mathrm{dL}(\mathrm{SD}=1.9)$, respectively. Lead workers had significantly lower ALAD activity then reference subjects $(42.6 \pm 22.4 \mathrm{U} / \mathrm{L}$ vs. $64.3 \pm 13.8 \mathrm{U} / \mathrm{L}, \mathrm{P}<0.001)$. According to the multiple regression results, the following independent variables were significant related to ALAD activity: ALAD activities in females were much lower $8.15 \mathrm{U} / \mathrm{L}$ then males $(\mathrm{P}<0.001)$; blood lead and glucose AC were inversely associated with ALAD activity $(\mathrm{P}<0.001)$, but the effect of blood lead was profound. The regression coefficients of blood lead and glucose AC were 1.04 and 0.11 , respectively. Individuals with alcohol consumption showed lower ALAD activity $(P=0.049)$. The possible threshold value of blood lead for ALAD activity was determined around $10 \mu \mathrm{g} / \mathrm{dL}$. ALAD activity was inhibited by lead sensitively and stoichiometrically, thus ALAD activity may be adopted as a reliable biomarker of lead toxicity in humen.
\end{abstract}

Key words: blood lead, delta-aminolevulinic dehydratase, prophobilinogen synthesis, ALAD activity, ALAD polymorphism, hemopoietic enzyme, hematologic system

\section{Introduction}

One of the mechanisms of lead induced anemia is, specifically, inhibition of heme synthesis. Lead inhibits three enzymes in the heme biosynthesis pathway- $\delta$-aminolevulinic acid dehydratase (ALAD), coporphyrinogen oxidase, and ferrochelatase- but its effects on ALAD are the most profound. Lead inhibits ALAD stoichiometrically, and the degree of erythrocyte ALAD inhibition has been used clinically to gauge the degree of lead poisoning (Chisolm et al., 1985; Jaffe et al., 1991). ALAD inhibition results in the buildup of aminolevulinic acid, detectable in the plasma and urine at blood lead levels more than $10 \mu \mathrm{g} / \mathrm{dl}$. At the molecular level, lead displaces a zinc ion at the metal binding site, not the active site, producing inhibition through a change in the enzyme's quaternary structure (Rogan et al., 1986; Warren et al., 1998). Thus, the ALAD activity may be inversely related to higher blood lead levels.

The goal of this study was to establish ALAD activity assay in Taiwan firstly. Secondly, we compared the frequency of ALAD genotype in occupational lead-exposure workers to general population, and find the genotype more vulnerable to environmental and occupational lead. Finally, the effects of lead exposure on hematological system and the ALAD activity with the modification of the relative genotypes were analyzed.

\section{Materials and Methods}


The study was carried out in a lead manufactory, We asked the workers whether they agreed to participating the study that checked their ALAD genotype and activity in addition to the regular health examination, and their consent form were obtained. On the other hand, age and sex matched workers from the same area but different plants without lead used in their industries were selected to serve as reference group; thus, their socioeconomic status was similar to the lead workers.

ALAD genotyping was conducted by PCR amplification, followed by polymorphism-specific restriction enzyme digestion and gel analysis. ALAD genotyping was conducted by using the polymerase chain reaction and restricted fragment length polymorphisms (PCR-RFLP). ALAD activity was measured using the European standardized method with spectrophotometric determination carried out at $555 \mathrm{~nm}$ (Berlin and Schaller, 1974).

Regression analysis could give us the correlation of ALAD activity and blood lead levels with adjustment of the other confounders. In addition, the effects of interaction between lead and ALAD activity or the other biochemical can be estimated by multiple regressions. Significance was set at 0.05 (two-tailed).

\section{Results and Discussion}

In this study, 229 ALAD1-1 homozygotes (96.2\%), 8 ALAD1-2 heterozygotes (3.8\%) were identified, and none of ALAD2-2 homozygote was observed. Blood lead levels in lead workers and reference subjects were $19.5 \mu \mathrm{g} / \mathrm{dL}(\mathrm{SD}=14.7)$ and $2.9 \mu \mathrm{g} / \mathrm{dL}(\mathrm{SD}=1.9)$, respectively. Lead workers had significantly lower
ALAD activity then reference subjects $(42.6 \pm 22.4 \mathrm{U} / \mathrm{L}$ vs. $64.3 \pm 13.8 \mathrm{U} / \mathrm{L}, \mathrm{P}<0.001)$. According to the multiple regression results, the following independent variables were significant related to ALAD activity: ALAD activity in females were much lower $8.15 \mathrm{U} / \mathrm{L}$ then males $(\mathrm{P}<0.001)$; blood lead and glucose AC were inversely associated with ALAD activity $(\mathrm{P}<0.001)$, but the effect of blood lead was profound. The regression coefficients of blood lead and glucose AC were 1.04 and 0.11 , respectively. Individuals with alcohol consumption showed lower ALAD activity $(\mathrm{P}=0.049)$. The possible threshold value of blood lead for ALAD activity was determined around $10 \mu \mathrm{g} / \mathrm{dL}$.

Table 1 Comparison on the characteristics of control and lead-exposed groups

\begin{tabular}{lccc}
\hline Characteristics & $\begin{array}{c}\text { Control } \\
\mathrm{n}=117\end{array}$ & $\begin{array}{c}\text { Lead-exposed } \\
\mathrm{n}=121\end{array}$ & $p$-value \\
\hline Age (year) & $41.7 \pm 11.8$ & $41.5 \pm 8.3$ & $0.916^{\mathrm{a}}$ \\
Working duration (year) & - & $11.7 \pm 5.3$ & - \\
Gender (Male \%) & $71(61.1 \%)$ & $98(81.0 \%)$ & $0.01^{\mathrm{b}}$ \\
BMI $\left(\mathrm{kg} / \mathrm{m}^{2}\right)$ & $24.9 \pm 4.1$ & $24.4 \pm 3.5$ & $0.310^{\mathrm{a}}$ \\
Blood lead $(\mu \mathrm{g} / \mathrm{dL})$ & $2.9 \pm 1.8$ & $19.7 \pm 14.7$ & $<0.001^{\mathrm{a}}$ \\
Hemoglobin (mg/dL) & $14.7 \pm 1.5$ & $14.9 \pm 1.4$ & 0.168 \\
Hct $\%$ ) & $43.5 \pm 3.8$ & $44.4 \pm 3.5$ & $0.060^{\mathrm{a}}$ \\
ALAD activity (U/L) & $63.32 \pm 14.01$ & $41.58 \pm 22.07$ & $<0.001^{\mathrm{a}}$ \\
ALAD 1-1 type (\%) & $113(96.6 \%)$ & $116(95.9 \%)$ & $0.773^{\mathrm{a}}$ \\
ALAD 1-2 type (\%) & $4(3.4 \%)$ & $5(4.1 \%)$ & \\
Glucose AC (mg/dL) & $106.7 \pm 38.6^{\dagger}$ & $101.8 \pm 32.3$ & $0.309^{\mathrm{a}}$ \\
Glucose AC $\geqq 126(\%)$ & $8(6.8 \%)$ & $9(7.4 \%)$ & $0.857^{\mathrm{b}}$ \\
Alcohol use twice/week (\%) & $9(7.7 \%)$ & $32(26.4 \%)$ & $<0.001^{\mathrm{b}}$ \\
Smokers $(\%)$ & $16(13.7 \%)$ & $49(40.5 \%)$ & $<0.001^{\mathrm{b}}$ \\
\hline${ }^{\mathrm{a}}$ T-test & & & \\
${ }^{\mathrm{b}}$ Chi-Square test & & & \\
${ }^{\dagger}$ valid of 104 subjects in control group &
\end{tabular}

Table 2 Multiple linear regression model for ALAD activity

\begin{tabular}{|c|c|c|c|c|}
\hline \multirow[b]{2}{*}{ Variables } & \multicolumn{2}{|c|}{ All $(n=238)$} & \multicolumn{2}{|c|}{ ALAD 1-1 type (n=229) } \\
\hline & $\begin{array}{l}\text { Regression } \\
\text { coefficient }\end{array}$ & $p$-value & $\begin{array}{l}\text { Regression } \\
\text { coefficient }\end{array}$ & $p$-value \\
\hline Constant & $83.84(6.76)$ & $<0.001$ & $82.86(6.91)$ & $<0.001$ \\
\hline ALAD type (1-2 vs $1-1)$ & $-4.12(4.75)$ & 0.39 & \multicolumn{2}{|l|}{--} \\
\hline Gender (M vs $F$ ) & $-8.08(2.27)$ & $<0.001$ & $-8.28(2.31)$ & $<0.001$ \\
\hline Age (year) & $-0.16(0.09)$ & 0.08 & $-0.19(0.09)$ & 0.05 \\
\hline $\mathrm{BMI}\left(\mathrm{kg} / \mathrm{m}^{2}\right)$ & $0.20(0.26)$ & 0.45 & $0.33(0.27)$ & 0.23 \\
\hline Blood lead $(\mu \mathrm{g} / \mathrm{dL})$ & $-1.04(0.08)$ & $<0.001$ & $-1.05(0.08)$ & $<0.001$ \\
\hline Glucose AC (mg/dL) & $-0.11(0.03)$ & $<0.001$ & $-0.12(0.03)$ & $<0.001$ \\
\hline Smoking (yes/ no) & $0.96(2.37)$ & 0.68 & $1.22(2.40)$ & 0.61 \\
\hline \multirow[t]{2}{*}{ Drinking (yes/ no) } & $-5.47(2.69)$ & 0.04 & $-5.51(2.70)$ & 0.04 \\
\hline & \multicolumn{2}{|c|}{$\left(\operatorname{adj~} R^{2}=0.639\right)$} & \multicolumn{2}{|c|}{$\left(\operatorname{adj} R^{2}=0.644\right)$} \\
\hline
\end{tabular}

\footnotetext{
${ }^{*} p$-value $<0.05,{ }^{* *} p$-value $<0.01,{ }^{* * *} p$-value $<0.001$
} 


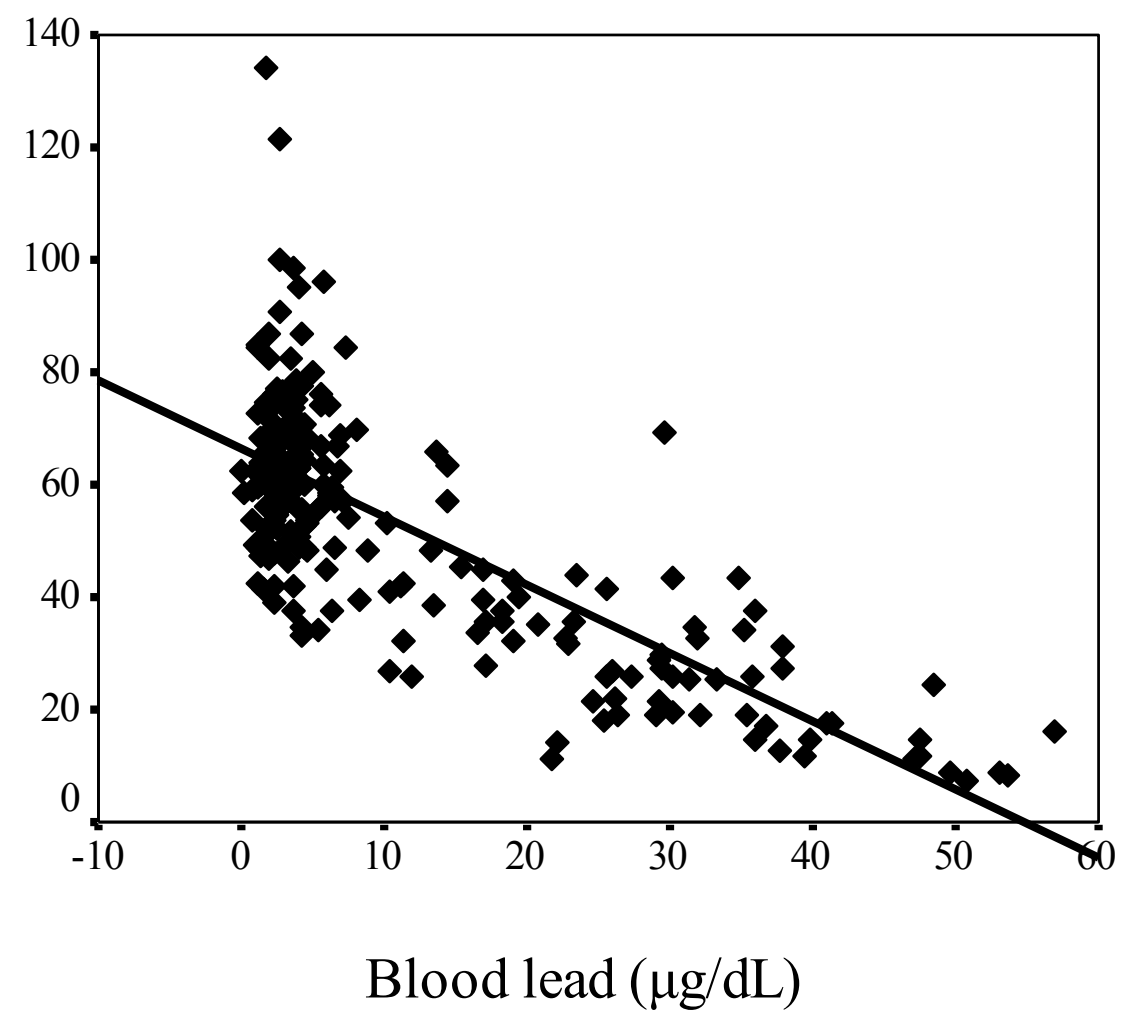

Fig. 1. Linear regression trend between ALAD activity and blood lead in all subjects. $(n=238, R 2=0.58, p<0.001)$

\section{Conclusion}

ALAD activity was inhibited by lead sensitively and stoichiometrically, thus ALAD activity may be adopted as a reliable biomarker of lead toxicity in human.

\section{Acknowledgements}

The authors thank the financial supports providing from Taiwan National Science Council (Project NSC96-2314-B-037-049) .

\section{References}

Berlin A, Schaller KH. European standardized method for the determination of delta-aminolevulinic acid dehydratase activity in blood. Z Klin Chem Klin Biochem 1974; 12: 389-90.
Chisolm JJ, Jr., Thomas DJ, Hamill TG. Erythrocyte porphobilinogen synthase activity as an indicator of lead exposure in children. Clin Chem 1985; 31: 601-5.

Jaffe EK, Bagla S, Michini PA. Reevaluation of a sensitive indicator of early lead exposure. Measurement of porphobilinogen synthase in blood. Biol Trace Elem Res 1991; 28: 223-31.

Rogan WJ, Reigart JR, Gladen BC. Association of amino levulinate dehydratase levels and ferrochelatase inhibition in childhood lead exposure. J Pediatr 1986; 109: 60-4.

Warren MJ, Cooper JB, Wood SP, Shoolingin-Jordan PM. Lead poisoning, haem synthesis and 5-aminolaevulinic acid dehydratase. Trends Biochem Sci 1998; 23: 217-21. 